\title{
The cloud logistics modeling and validation based on $\mathrm{Pi}$ calculus XueFeng $\mathrm{He}^{1}$, JiuLei Jiang ${ }^{2}$ and GuangHui Wei ${ }^{3}$
}

College of Computer Science and Engineering, Beifang University of Nationalities, Yinchuan 750021, China

1994029@nun.edu.cn

Keywords: Cloud logistics, Interactive model, BPMN2.0, Pi calculus

\begin{abstract}
Following many bottlenecks of the development of the logistics industry at present,a interactive model of cloud logistics is proposed in this paper.Describing the interactive model of cloud logistics by BPMN2.0 choreography,then the Pi calculus is adopted to make rigorous formal definition for the basic activities and structured activity of the BPMN2.0, we use the validation tool MWB (mobile work platforms) of Pi calculus to simulate and to ensure the correctness of the information transmission in the organization's internal or among the organizations to provide reliable interactive model of business process for the system development in future.
\end{abstract}

\section{Introduction}

With the rapid development of electronic commerce, the influence of the logistics is more and more obvious to e-commerce.In a completed order business on online,logistics entity must fully meet the electronic commerce trade form and adapt to its rapid development.However,Logistics resources are difficult for uniforming distribution at present. Therefore, it is difficult to meet the demand of high speed and low cost logistics service for the reason of the high cost.

In view of the logistics development bottleneck from above, cloud logistics has brought new hope for the development of the logistics integration developed on the basis of cloud computing. All orders is the source of the water, cloud platforms handle orders information and classify the goods according to the time, location, and degree of emergency, orders are classified concentrated to be sent out by the appropriate transportation company.Express company access to the cloud logistics platform through a computer to obtain distribution information and complete distribution services. As a result, cloud logistics platform will be the trend of development in the future.Cloud logistics mode are introduced[1], Which links on cloud computing and Internet of things. studying the system framework of cloud logistics and cites a example of which the applications of freight enterprise based on cloud logistics. [2]And the paper also describe a development mode of cloud logistics, and according to the cloud logistics mode which is defined by NIST to put forward a cloud platform trading and logistics process in specific areas. The cloud computing method which can promote the logistics control is discussed and compared[3]. Cloud computing can provide key management software, effectively reduce the maintenance cost of hardware and software, and study the cold chain logistics system that analysis and design this system which based on cloud computing platform[4].This paper analyzed the working principle and characteristic of the cloud logistics [5], studied one-stop service platform which based on cloud logistics, the platform can provide comprehensive, rapid, efficient logistics service for supply chain users. [6]And also analyzed the cloud logistics platform is a good solution for the construction of modern public logistics information platform.[7] put forward the construction of logistics public information platform through the enterprise cooperation, and analyze the corresponding operation mode, [8] survey and analyze the challenges and benefits of cloud platform about the third party logistics company. They combine on the logistics distribution business of agricultural products and then exemplify and analyse the implementation process of cloud logistics which is based on the architecture[9].

What has been discussed above analyzed the development present situation and trend of development of clouds logistics, However we lack the business process modeling tools to describe 
cloud complete logistics research. Currently,while BPMN [10]as a standard in most current process modeling tool manufacturers, combined with PI calculus to verify the BPMN model, so as to verify the accuracy of business processes. Pi calculus is a kind of process algebra that has the strong power of expression and simple form. Compared with other formal methods, Pi calculus also have the ability to transport channel, so it has an advantage to describe the system of the mobility. So This paper first describes the business flow of cloud logistics by BPMN, and then verify the interaction processes of cloud logistics by using the formal method of Pi calculus.

\section{The basis of Relevant theoretical}

\subsection{BPMN}

BPMN (Business Process Modeling Notation) is a symbols standard to describe the Business Process, it can be easily understanded and communicated between business personnel and designers.

2.2 Pi calculus

$\mathrm{Pi}$ calculus originated in the late 1980s that was put forward by the Turing winner Robin Milner,it is a kind of calculus model to describe and analyze the concurrent systems, name is the most basic elements in the $\mathrm{Pi}$ calculus and it has infinite number,Tags: $\mathrm{x}, \mathrm{y}, \ldots \in$ Name;Another concept is the process, The process expression consists of name by means of the subordinate grammar (BNF, EBNF):

Process $\mathrm{P}::=\mathrm{M}|\mathrm{P}| \mathrm{Q}|! \mathrm{P}|(\mathrm{vx}) \mathrm{P}$

$\mathrm{P} \mid \mathrm{Q}$ is called parallelism,it is interpreted as process independent parallel execution and can communicate by the channel between $\mathrm{P}$ and $\mathrm{Q}$; !P is called the replication, just as many process parallel execution at the same time: $\mathrm{P}|\mathrm{P}| \ldots$, There is no limit on the number, generated dynamically according to need; $(v \mathrm{x}) \mathrm{P}$ is called restriction or new name, name $\mathrm{x}$ is effective only in the process $\mathrm{P}$.

\section{Analysis of Cloud logistics process}

\subsection{Explore the cloud logistics platform}

Logistics process includes packing, loading and unloading, transportation, storage, distribution processing, distribution and logistics information etc.Firstly the logistics operation is shouldered mainly by manufacturing enterprises themselves, namely, the 1PL and the 2PL; with the development of social economy, the logistics of high cost and low efficiency that is difficult to meet consumer demand, and then the 3PL and the 4PL appear here, in fact, they recombine logistics activities, namely business reconfiguration, it implements the specialized service activities. The following figure 1 describes the traditional logistics process.

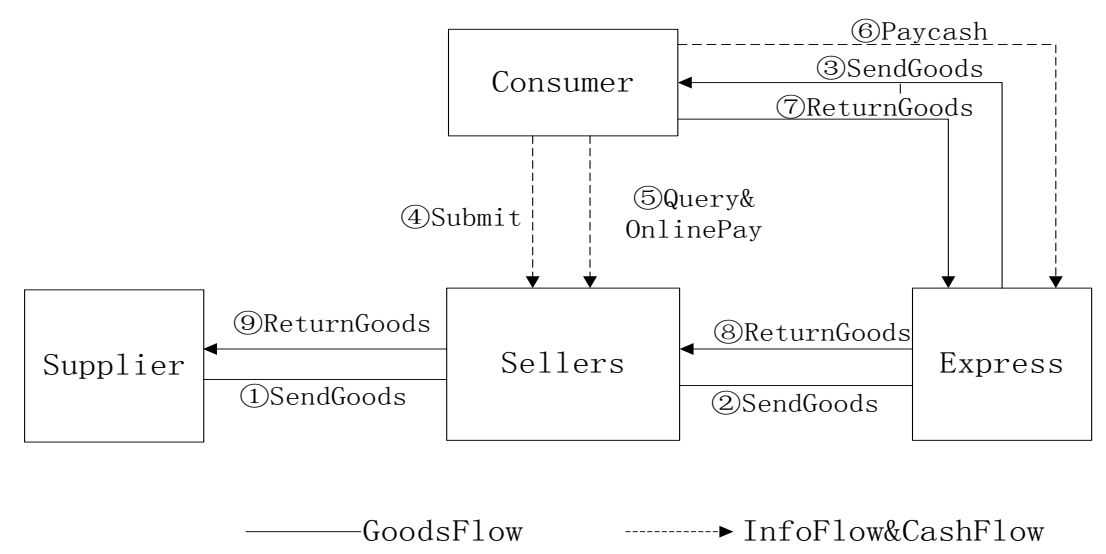

Fig.1 Traditional logistics process

Pictured above is the general logistics flow of electricity enterprise,third party logistics 
enterprises have been circulated as an important link, this model can not effectively use of resources of third party logistics to reduce costs on both sides of the logistics.

With the development of cloud computing, cloud computing provides a comparatively perfect right business reconfiguration and information sharing platform for the development of logistics. Based on B2C e-commerce logistics as an example, this paper analyses the business process of cloud logistics, as shown in the following Fig.2.

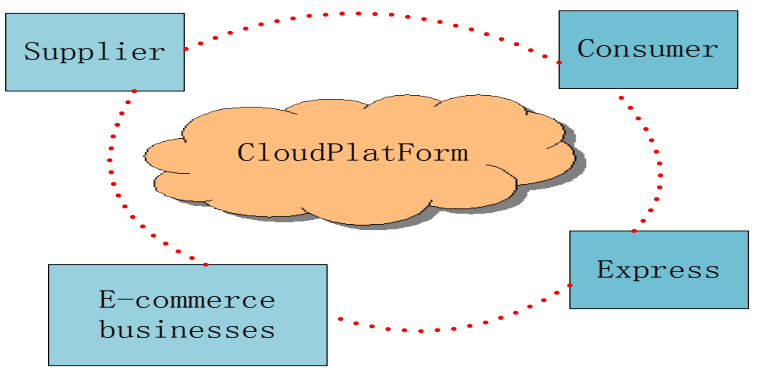

Fig.2 Mode of cloud logistics

According to the above figure, we know that all the resources of the electronic commerce has been integrated into the cloud platform, business interactions by an optimal way,In order to achieve high efficiency and low cost service.

3.2 Describe the flow chart of cloud logistics by BPMN2.0

Accept-delivery process in the B2C e-commerce enterprise based on cloud logistics platform.we regard the electricity business enterprise as a seller and the receiving party clients as the buyer, The related information of each link in the process of the clouds logistics which integrated into the cloud logistics platform. The specific process as shown in Fig.3.

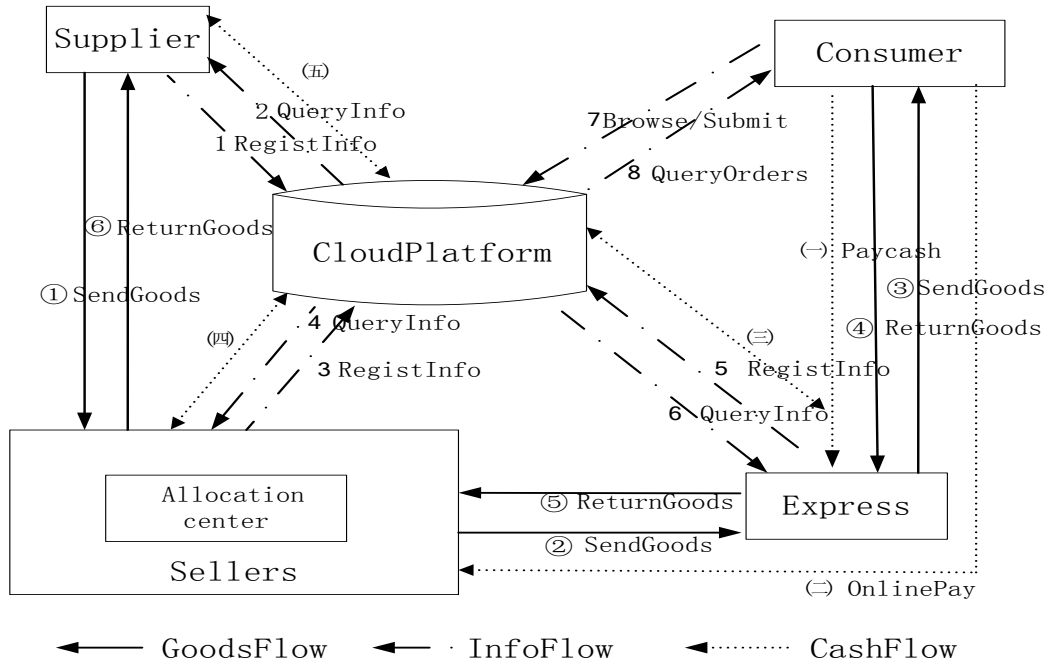

Fig.3 Business flow chart of cloud logistics platform

In the figure above, Serial number 1-8 representes information flow ; Serial number (1) 6represent physical flow; ( - - (五)shows the flow of funds;

In the process of cloud logistics, including five roles: Consumer, Cloud platform, Cellers,Express,Supplier.firstly,supplier provide the goods to the sellers, sellers choose the most convenient delivery company to put the goods into the hands of customers, the business process is over until the customer payment;

BPMN collaboration diagrams of the business process as shown in Fig.4: 


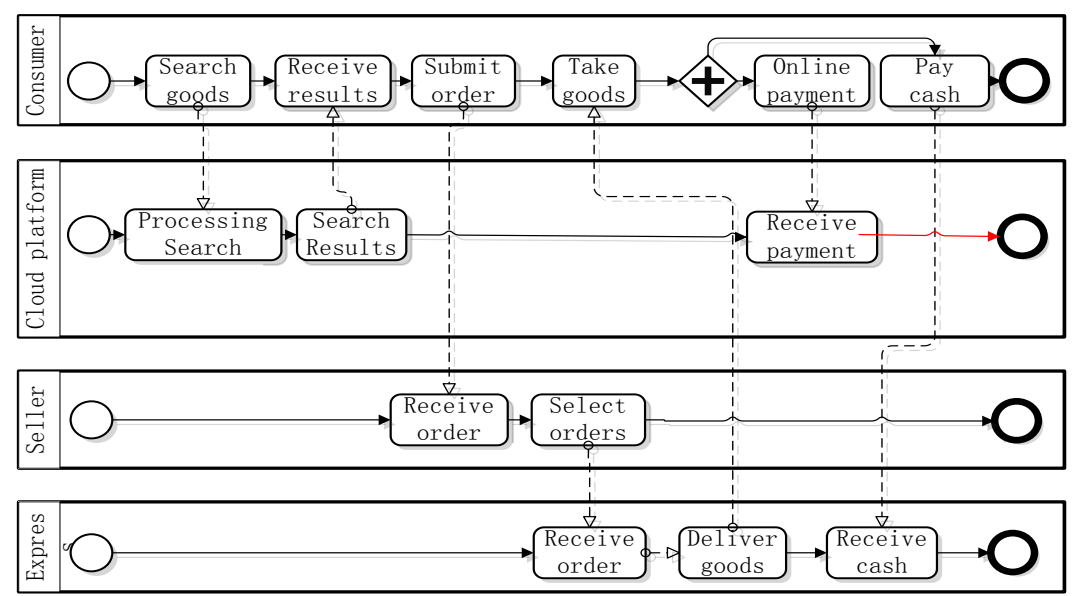

Fig.4 BPMN collaborative flow chart for order interaction

Business interaction is described by BPMN2.0 in the diagram above, but the BPMN can't formal analysis for choreography, so the following we describe the pattern of choreography by Pi calculus to validate the correctness of the model further.

3.3 Pi calculus describing the choreography of BMPN2.0

Here are a few definitions on the basis of business process diagrams:
Definition
1
Message
in the
interaction :

New $=\{$ registInfo, queryInfo,browse,result,submit,dispatch,queryOrders,sendGoods,payCash, onlinePay, returnGoods \};

RegistInfo: Supplier, Sellers, Express and other departments release own supply and demand information in the cloud logistics platform ; QueryInfo: each department query information through the cloud logistics platform; Browse: Browse commodity; Result: the goods that are selected by user; Submit: Submit orders; Dispatch: express company send goods; QueryOrders: real-time query order; SendGoods: delivery; PayCash: cash payment; OnlinePay: online payment; ReturnGoods: return the goods.

Definition 2 the set of transport channels between different departments: Channels $=\{$ sg,sr,so,seo,dg,op,pc $\}$

The corresponding relation of each channel in the following table:

Table 1: the corresponding channel between different departments

\begin{tabular}{|l|l|l|l|l|}
\hline $\begin{array}{l}\text { Department } \\
\text { name }\end{array}$ & Consumer & ClouldPlatform & Sellers & Express \\
\hline Consumer & $\mathrm{c}$ & $\mathrm{sg}, \mathrm{sr}$ & so & $\mathrm{dg}, \mathrm{pc}$ \\
\hline ClouldPlatform & $\mathrm{sg}, \mathrm{sr}$ & $\mathrm{cp}$ & null & null \\
\hline Sellers & $\mathrm{so}$ & null & $\mathrm{s}$ & seo \\
\hline Express & $\mathrm{dg}, \mathrm{pc}$ & null & seo & $\mathrm{e}$ \\
\hline
\end{tabular}

In Table 1, "null” represents that there was no interaction between departments, letter c,cp,s and e mark respectively the first letters of the four department name that instructs the channels of news transmission within the department, if the news of transmission are much more within the department, we mark through using internal name combined with Arabic numerals. Such as consumer, the set of internal transport channel: $C=\{c 1, c 2, c 3, c 4, c 5\}$.we get Pi calculus model according to the above definition of consume:

$$
\begin{aligned}
& \text { def } \\
& \text { consumer }=\overline{s g}\langle\text { browse }\rangle . \overline{c 1}\langle m s g\rangle \mid c 1\langle m s g\rangle . s r\langle\text { result }\rangle . \overline{c 2}\langle m s g\rangle \\
& \mid c 2\langle m s g\rangle . \overline{s o}\langle\text { submit }\rangle . d g\langle\text { sendGoods }\rangle . \overline{c 3}\langle m s g\rangle \mid c 3\langle m s g\rangle . \\
& (\overline{o p}\langle\text { onlinePay }\rangle . \overline{c 4}\langle m s g\rangle \mid \overline{p c}\langle\text { payCash }\rangle \cdot \overline{c 5}\langle m s g\rangle)((c 4\langle m s g\rangle \mid c 5\langle m s g\rangle) .0
\end{aligned}
$$


The Pi calculus model of cloudPlatform:

cloudplatform $\stackrel{\text { def }}{=} \mathrm{sg}\langle$ browse $\rangle . \tau . \overline{s r}\langle$ result $\rangle .0$

Pi calculus model of Sellers:

seller $\stackrel{\text { def }}{=}$ so $\langle$ submit $\rangle . \tau . \overline{\text { seo }}\langle$ dispatch $\rangle$. op $\langle$ onlinePay $\rangle .0$

The Pi calculus model of express company:

express $\stackrel{\text { def }}{=} \operatorname{seo}\langle$ dispatch $\rangle \cdot \tau \cdot \overline{d g}\langle$ sendGoods $\rangle \cdot \mathrm{pc}\langle$ payCash $\rangle .0$

$\mathrm{Pi}$ calculus model of the whole system is as follow:

consumer(sg, sr, c1, c2, c3, c4, c5) $\stackrel{\text { def }}{=} \tau \cdot \overline{s g}\langle$ browse $\rangle . \overline{c 1}\langle m s g\rangle \mid c 1\langle m s g\rangle . s r\langle$ result $\rangle . \overline{c 2}\langle m s g\rangle$

$\mid c 2\langle m s g\rangle . \overline{s o}\langle$ submit $\rangle . d g\langle$ sendGoods $\rangle . \overline{c 3}\langle m s g\rangle \mid c 3\langle m s g\rangle$.

$(\overline{o p}\langle$ onlinePay $\rangle . \overline{c 4}\langle m s g\rangle \mid \overline{p c}\langle$ payCash $\rangle \cdot \overline{c 5}\langle m s g\rangle) \mid(c 4\langle m s g\rangle \mid c 5\langle m s g\rangle)$. .consumer(sg, sr, c1, c2, c3, c4, c5)

cloudplatform $(\mathrm{sg}, \mathrm{sr}) \stackrel{\text { def }}{=} \mathrm{sg}\langle$ browse $\rangle \cdot \tau \cdot \overline{\mathrm{sr}}\langle$ result $\rangle \cdot \operatorname{cloudplatform}(\mathrm{sg}, \mathrm{sr})$

seller(so, seo, op) $\stackrel{\text { def }}{=}$ so $\langle$ submit $\rangle . \tau . \overline{s e o}\langle$ dispatch $\rangle$. op $\langle$ onlinePay $\rangle . s e l l e r($ so, seo, op)

$\operatorname{express}(\mathrm{seo}, \mathrm{dg}, \mathrm{pc} \stackrel{\text { def }}{=} \operatorname{seo}\langle$ dispatch $\rangle \cdot \tau \cdot \overline{\mathrm{dg}}\langle$ sendGoods $\rangle \cdot \mathrm{pc}\langle$ payCash$\rangle$.express (seo, dg, pc)

\section{Process validation Based on the MWB}

This section detectes the process with the help of a mobile work platform (MWB) command, here, we only provide example:the interaction between the consumer and cloudpaltform process and to detectes accuracy of the interactive process,similarly,we can detect the accuracy about the processes of other participants. The process expression of the consumer and the cloudplatform that converts into MWB coding by the aid of the corresponding rules of the calculus convert to MWB coding, coding is as follows:

agent $\operatorname{CON}($ sg,sr,browse,msg,result)='sg<browse $>$.sr(result).CON $<$ sg,sr,browse,msg,result $>$

agent $\quad$ CLOU(sr,sg,browse,result) $=$ sg (browse). 'sr $<$ result $>$.CLOU $<$ sr, sg, browse,result $>$

agent $\mathrm{P}$ (sg,browse,msg,sr,result) $=\mathrm{CON}<$ sg,sr, browse, msg,result $>\mid \mathrm{CLOU}<$ sr,sg, browse, result $>$ Then start the platform, the concrete steps and display the results as follows:

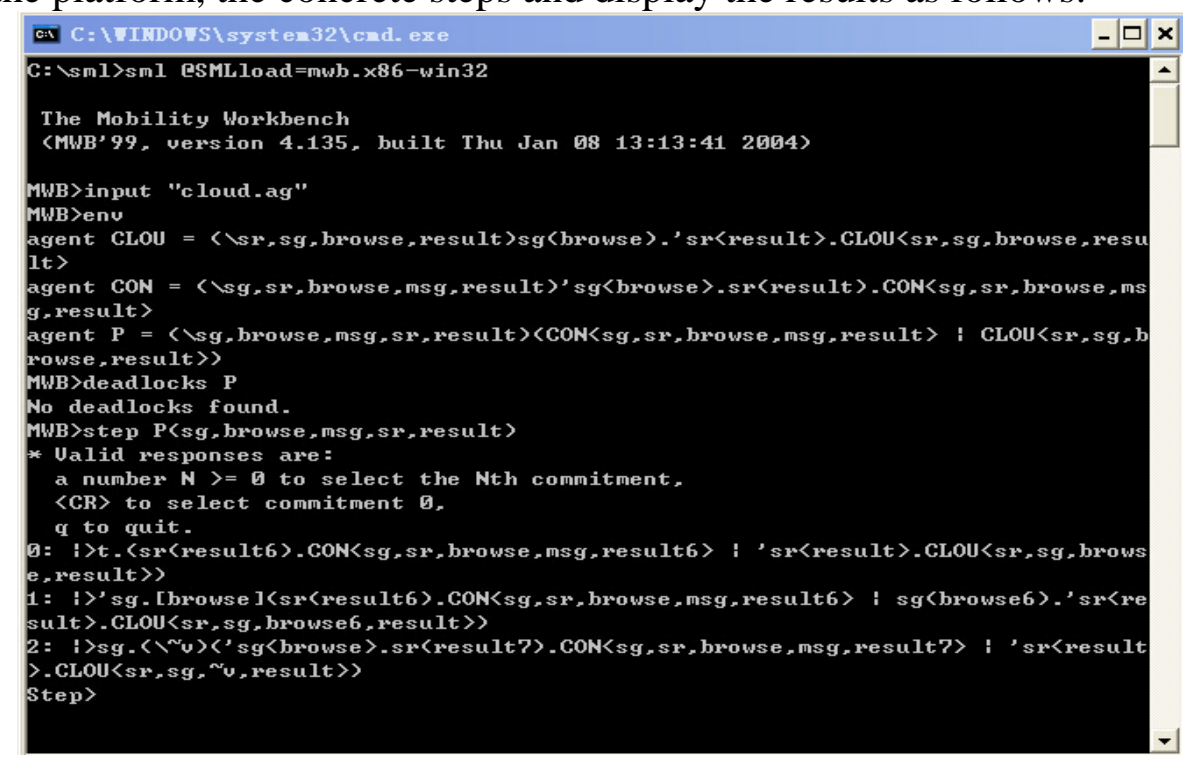

Fig.5 The first step of 0 path of simulation $\mathrm{P}$ agent

Step one: input “input "loud.ag"”:import the source file (the source file is stored in the MWB root directory).

Step 2: Input “env": get all the agents.

Step 3: Verify the deadlock, input "deadlocks P", verify whether there is a deadlock process of interaction, the results of display in the process:" No deadlocks found", it illustrates the correctness of the interaction of process between agents. 
Step 4: Enter “step $\mathrm{P}<\mathrm{sg}$, msg, sr, result>”, it can simulate the interaction process of $\mathrm{P}$ agent, shows all possible paths in the process of interaction, and then choose serially the same path that can simulate each interaction steps, as shown in figure 8, after it carried out the first step interaction, agent CON sends information "msg" by channel sr,agent CLOU receives the information by the channel sg, and shows the next interaction agent, CLOU send information "result" through channel sr, agent CON receives information "result". There are three kinds of path selection, and then

choose a 0 path again, get the following results:

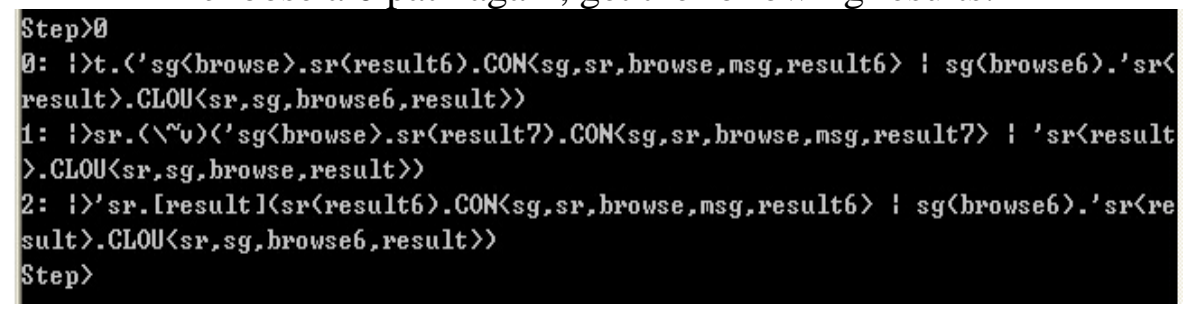

Fig.6 The second step of 0 path of simulation P agent

Get the following results through third 0 transfer:

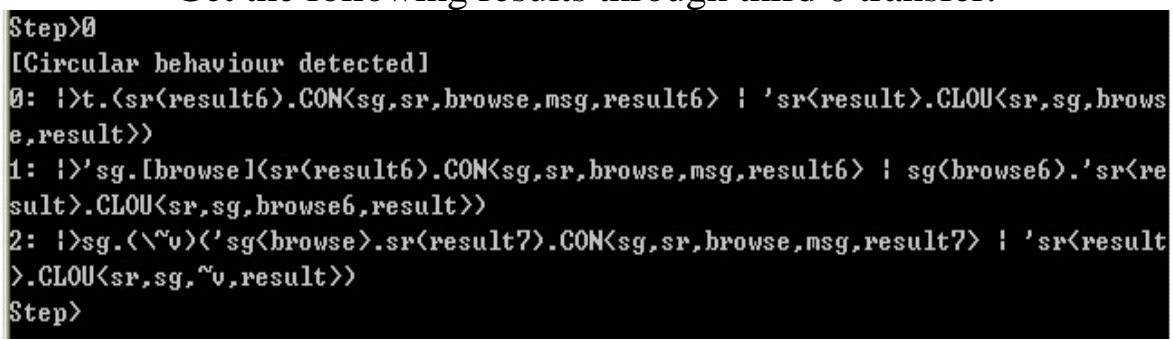

Fig.7 The third step of 0 path of simulation $P$ agent

Here are the same result by comparing Fig.7 and Fig.5, and show the "Circular behaviour detected" that tells the first interaction has been over, the second process interaction will start.

\section{Summary}

This paper analyzes and summarizes the development status of current cloud logistics and proposes a model cloud logistics and describes the main cloud logistics business process with the aid of BPMN, and describes the interactions of main business processes by means of the Pi calculus, finally by the aid of MWB, we provide an example that could verify the process does not exist deadlock between the consumer and cloud platform, and every step is simulated in the interaction process, and verify the correctness of process. we can realize the cloud logistics system with the aid of JBPM in the next research to provide tentative implementation of process for the cloud logistics development, and make further study of the software system, which includes a series of process about the business process description to the implementation of system.

\section{Acknowledgments}

This work was supported by project of National Natural Science Foundation, China (No.61162013), and NSFC pre-breeding program of Beifang University of Nationalities (No. 2012QZP02).

\section{References}

[1] Qifeng W, Hongbo L, Yu J. Study on Cloud Logistics System Framework and Application Mode [J][J]. Telecommunications Science, 2012, 3: 024.

[2] Holtkamp B, Steinbuss S, Gsell H, et al. Towards a logistics cloud[C]//Semantics Knowledge and Grid (SKG),2010 Sixth International Conference on. IEEE, 2010: 305-308.

[3] Schuldt A, Hribernik K, Gehrke J D, et al. Cloud Computing for Autonomous Control in Logistics[C]//GI Jahrestagung (1).2010: 305-310. 
[4] Li X, Wang Y, Chen X. Cold chain logistics system based on cloud computing[J]. Concurrency and Computation: Practice and Experience,2012,24(17): 2138-2150.

[5] Wang X, Li W, Zhong Y, et al. Research on cloud logistics-based one-stop service platform for logistics center[C]//Computer Supported Cooperative Work in Design (CSCWD), 2012 IEEE 16th International Conference on. IEEE, 2012: 558-563.

[6] JIANG Y, LI X, TAN H. Logistics Network System Construction Based on Cloud Computing Processing[J]. Journal of Guangdong University of Education, 2013, 3: 014.

[7] Li C, Zhang X, Li L. Research on Comparative Analysis of Regional Logistics Information Platform Operation Mode Based on Cloud Computing1[J]. International Journal of Future Generation Communication \& Networking,2014, $7(2)$.

[8] Gantzia D, Sklatinioti M E. Cloud computing in the 3PL industry. A profound insight into the benefits \& challenges of cloud-based services: A two fold approach[J]. 2014.

[9] ZHENHAN X L. StudyonServiceModel of Agricultural Product Logistics Based on Framework of Cloud Logistics[J].The Conference on WEB Based Business Management, 2012(2012): 81-85.

[10] Model B P. Notation (BPMN), Version 2.0, Object Management Group, Jan. 2011[J]. last accessed: 14.03. 13, 2013. 